\title{
High-Precision UWB Based Localisation for UAV in Extremely Confined Environments
}

\author{
Beiya Yang, Student Member, IEEE, Erfu Yang, Member, IEEE, Leijian Yu and Andrew Loeliger
}

\begin{abstract}
In this paper, a high-precision ultra-wideband (UWB) based unmanned aerial vehicle (UAV) localisation approach is proposed for applications in extremely confined environments. It is motivated by the emerging demand on autonomous inspection in such environments that are hard or impossible for humans to access. Instead of the traditional localisation techniques such as global positioning system (GPS), vision based or other localisation techniques, the UWB based localisation technique is adopted for precise UAV positioning due to its high accuracy, implementation simplicity and suitability in such environments. To avoid the requirement on strict synchronisation between sensor nodes and provide decimetre-level accuracy, the proposed algorithm combined the two-way time-of-flight (TW-TOF) localisation scheme with the maximum likelihood estimation (MLE) method. This differs from applications in other environments, the number and deployment area of anchor nodes are highly restricted in such environments. Therefore, an in-depth investigation for the anchor deployment

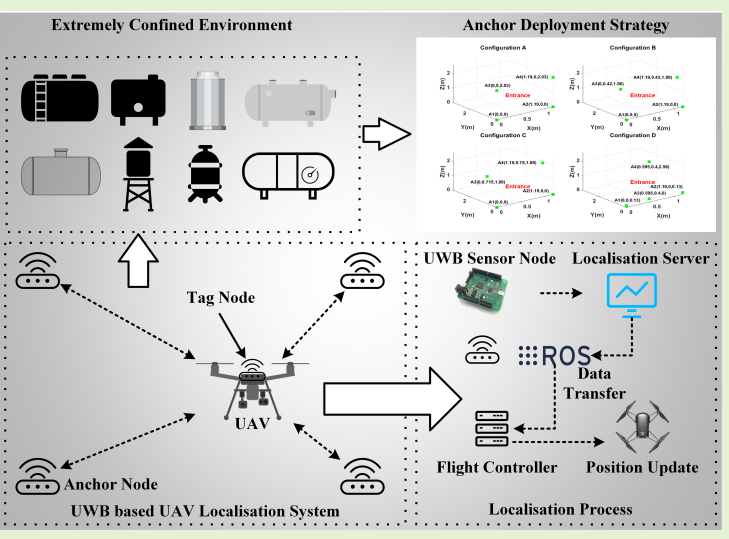
strategies is presented to find the most suitable geometry configurations with accurate and robust performance. Finally, extensive simulations, static experiments and flight tests have been conducted to validate the localisation performance under different deployment strategies. The experiments show that average localisation error and standard deviation (STD) under $0.2 \mathrm{~m}$ and $0.07 \mathrm{~m}$ are obtainable by using our proposed approach under three different geometry configurations of anchor nodes. This is suitable for different applications in extremely confined environments.
\end{abstract}

Index Terms-3D Localisation, Ultra-wideband (UWB), Unmanned Aerial Vehicle (UAV), Extremely Confined Environments, Anchor Deployment Strategy

\section{INTRODUCTION}

A LONG with increasing demands on industrial applications, leveraging unmanned aerial vehicles (UAVs) for the autonomous inspection in extremely confined environments such as the small storage tank or pressure vessel where it is normally unsafe and hard for humans to access becomes a new research direction [1], [2]. The commercial off-theshelf (COTS) product named Elios 2 [3] has already been released by Flyability and widely utilised on smart inspection in confined environments. Due to the satellite signal block, global positioning system (GPS) will lose its effectiveness in such environments. To overcome this, instead of the precise UAV positioning, a protective frame was mounted on Elios 2 for collision avoidance. However, without the precise position information, the flight of UAV can only rely on the manual control of the well-trained professionals. Besides, the high system cost (approximately $£ 27,000$ ) and instability of UAV

This work was supported in part by the UK Oil \& Gas Technology Centre under Grant No. Al-P-028 and in part by the University of Strathclyde (Corresponding author: Erfu Yang)

The authors are with the Department of Design, Manufacturing and Engineering Management, University of Strathclyde, 75 Montrose Street, Glasgow G1 1XJ, UK (e-mail: beiya.yang@strath.ac.uk; erfu.yang@strath.ac.uk; leijian.yu@strath.ac.uk; andrew.loeliger@strath.ac.uk). in such environments may extremely limit the application scenarios of the system. Consequently, precise, accurate and low-cost UAV positioning is still required in this area and will be the one key requirement for current applications.

Currently, numerous indoor localisation techniques have been investigated on UAV positioning in extremely confined environments. Among them, vision [4]- [6] and light detection and ranging (LiDAR) [7]- [12] based localisation techniques are known as the much-sought techniques in this area, owing to the enhanced localisation performance. For vision based techniques, Krul et al. [5] proposed a visual simultaneous localisation and mapping (VSLAM) based system which achieved precise positioning of the DJI Tello for indoor livestock and farming. However, the communication delay and low-visibility condition will all have the huge impact on the localisation performance. To overcome the performance degradation in the low-visibility condition, another UAV localisation system was designed in [6], which achieved high accuracy UAV positioning in dark and confined environment by leveraging the visual inertial odometry and the detection of existing known structural 3D landmarks. However, the prior information about 3D structural landmarks may restrict applications in unknown or difficult access environments. Different from vision based localisation techniques, the low-visibility 
impact can be ignored with LiDAR based approaches. A UAV navigation system was proposed by Wang et al. [7] which achieved robust and precise 3D UAV positioning with two additional laser scanners in the confined environment. However, the system still requires the prior information about the working environment and coordinates of its key corner features. Similarly, authors in [8] also designed a laser scanner based UAV positioning and navigation system focused on the smart inspection in a dark power plant boiler. Yet, the increasing energy consumption leads by the additional laser scanner may reduce the UAV operation time. Furthermore, authors in [9]- [12] all utilised LiDAR based localisation techniques as the main components to achieve precise UAV positioning in confined environments for smart inspection, classification and disaster management. Nevertheless, the weight, high energy consumption and cost for the LiDAR based system turn into an inevitable problem when designing the UAV system. This runs counter to applications on mini-UAVs or micro aerial vehicles (MAVs) in extremely confined environments, such as the small storage tank or pressure vessel. In order to tackle the aforementioned issues to achieve low energy consumption, low-cost and precise UAV positioning in extremely confined environments, the ultra-wideband (UWB) based localisation technique is introduced.

As depicted in Fig.1, the structure of the UWB based localisation approach is given. Leveraging the communication between low-cost sensor nodes, precise UAV positioning can be achieved. Low-visibility condition can be ignored due to the inherent nature of the radio frequency (RF) signal. Each sensor node is able to power itself with its on-board battery which has no impact on the operation time of UAV. In addition, with ultra-bandwidth, the system is more robust to the multipath effects and high temporal resolution can be achieved [13]. This makes it possible for precise and robust localisation with time-based localisation schemes in extremely confined environments. Time-of-arrival (TOA), time-of-flight (TOF) and time-difference-of-arrival (TDOA) are the typical representatives of time-based localisation schemes. For TOA and TOF based localisation schemes, the timestamps recorded by UWB sensor nodes from the transmitter and receiver are all required to find the intersection between a set of spheres for localisation. Therefore, the strict clock synchronisation between every sensor node is needed [14], [15]. For TDOA, only the timestamps from receivers are sufficient for positioning, but the clock synchronisation between anchor nodes (auxiliary nodes with known position) is still required [16][18]. To avoid the requirement of clock synchronisation, a two-way time-of-flight (TW-TOF) localisation scheme has been proposed, where the clock difference can be subtracted theoretically with TOA and time-of-departure (TOD) from both sides. As a result, plenty of research focused on UWB based UAV positioning in indoor or confined environments with TW-TOF localisation scheme has been carried out in the past few years [19]- [24].

Benini et al. [19] proposed a sensor fusion approach which achieved accurate localisation of mini-UAV in indoor environments with the combination of UWB, inertial measurement unit (IMU) and vision based technique. In [20], a UWB based

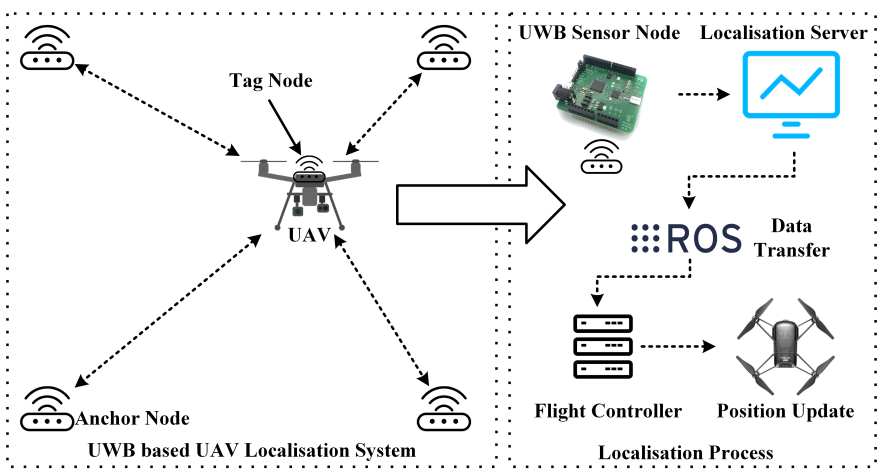

Fig. 1. Localisation structure of the UWB based UAV localisation approach.

UAV localisation solution was proposed which exploited TWTOF with extended Kalman filter (EKF) successfully obtained decimetre-level accuracy for UAV in indoor environment. Similarly, high accuracy localisation of UAV was achieved with TW-TOF through the fusion of UWB and IMU in [21][23]. However, applications in [20]- [22] were all focused on the relatively large and open environment, and the anchor layout in [19] and [23] was not addressed. Most relevantly, authors in [24] proposed a UWB based localisation approach for UAV which considered the geometry configurations of anchor nodes. Whereas, only a few analysis and evaluation at fixed points were given. The impact of different anchor layouts on UAV positioning was not taken into account. Apart from applications on UAV in confined environments, authors in [25] designed a sensor fusion system integrated IMU and UWB for precise positioning of coal mine robots in underground coal mine, which is also relevant to our work. UWB sensor nodes in the system were utilised to provide the observation information (distance information) for position update. The anchor deployment strategy was analysed for the precise determination of the covariance. However, the in-depth discussion on the performance variation with different geometry configurations of anchor nodes was never taken into account. Different from the aforementioned researches, considering our applications in extremely confined environments, it is hard or impossible to deploy plenty of anchor nodes to cover the whole positioning area. Therefore, with the limited number and restricted deployment area, the geometry configuration of anchor nodes needs to be addressed for precise UAV positioning.

Towards this end, a high-precision UWB based UAV localisation approach is proposed in this paper, focusing on applications in extremely confined environments. To avoid the requirement of strict synchronisation between sensor nodes and provide high accuracy localisation performance, the TWTOF based localisation scheme with the maximum likelihood estimation (MLE) method is utilised. Considering the limited number and restricted deployment area of anchor nodes, geometric dilution of precision (GDOP) [26], [27] and root mean square error (RMSE) of the algorithm under different geometry configurations of anchor nodes are investigated to find out the most suitable layout for precise UAV positioning. Finally, the simulations for the algorithm and experiments with a commercial low-cost mini quadcopter - DJI Tello 


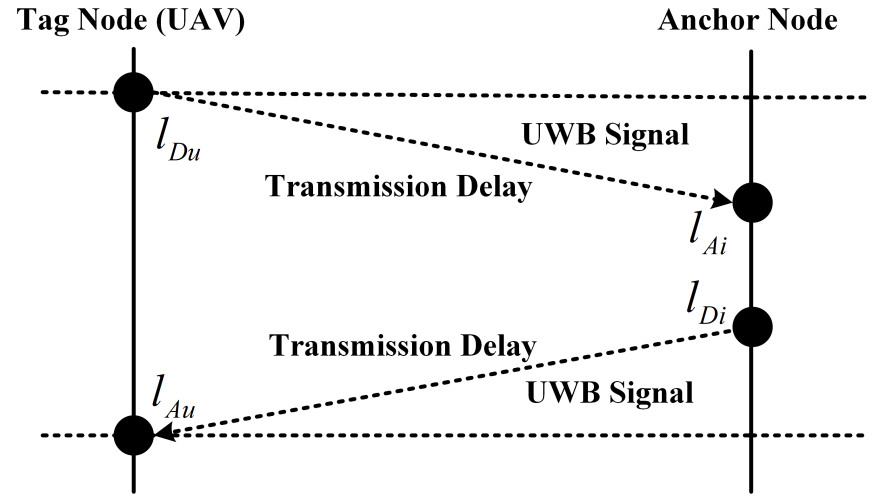

Fig. 2. TW-TOF based localisation scheme.

EDU have been conducted to verify the effectiveness of the algorithm in extremely confined environments and analyse the localisation performance under different anchor layouts. However, even with the existing characteristics of UWB based localisation techniques, the high-precision UAV positioning in extremely confined environments can be achieved, it still needs to note that, considering the inherent nature of the RF signal, the metallic environment will have huge impact on the communication between UWB sensor nodes, which may result in the localisation performance degradation or oscillation. Therefore, only the applications in non-metallic environments such as the storage tank or pressure vessel made of concrete, glass-reinforced plastic, thermoplastic or polyethylene are considered in this paper.

The rest of the paper is organised as follows. The overall architecture and details for the UWB based UAV positioning algorithm is described in Section II. Section III analyses the anchor deployment strategies with four different geometry configurations. The experiments part including the experiment setup and localisation performance evaluation is given in Section IV. Finally, the conclusion is drawn in Section V.

\section{UWB BASED UAV POSITIONING}

\section{A. Positioning Scheme}

In order to avoid the requirement of strict clock synchronisation between sensor nodes, and provide high accuracy performance, the TW-TOF based localisation scheme with UWB based localisation technique is exploited. It is clear from Fig.2, during the localisation process, the position request packet will be transmitted by the tag node equipped on UAV at time $l_{D u}$. Anchor node $i(i=1,2, \ldots, n)$ involved in this round will receive the request and record the timestamp as $l_{A i}$ after a transmission delay. After a response delay caused by the data processing, anchor node $i$ will respond to the request by transmitting back a response packet with the time departure stamp $l_{D i}$. Finally, the tag node will receive the response packet and record the TOA as $l_{A u}$ after a same transmission delay.

Assume that TOA and TOD for the communication signal between two sensor nodes be written as

$$
l_{A}=\tilde{l}_{A}+e,
$$

$$
l_{D}=\tilde{l}_{D}+e,
$$

where, $e$ is supposed to be the measurement noise of the UWB sensor node modelled as the additive white gaussian noise (AWGN) with zero mean and $\sigma_{e}^{2}$ variance [28], $\tilde{l}_{A}$ and $\tilde{l}_{D}$ represent the true value of TOA and TOD between tag node (equipped on UAV) and anchor node. Thus, according to the arithmetic theory of TW-TOF, the transmission delay between tag node and anchor node can be expressed as

$$
d_{i}=\varphi_{i} c=\left[\left(l_{A u}-l_{D u}\right)-\left(l_{D i}-l_{A i}\right)\right] c / 2 .
$$

In the equation, $d_{i}$ and $\varphi_{i}$ are defined as the measured distance and signal transmission delay between anchor node $i$ and UAV. $c$ is the velocity of electromagnetic wave. The equation can be further updated with the measurement noise $e$ from UWB sensor nodes,

$$
d_{i}=\left[\left(\tilde{l}_{A u}-\tilde{l}_{D u}\right)-\left(\tilde{l}_{D i}-\tilde{l}_{A i}\right)\right] c / 2+\omega_{i} .
$$

Within the equation, $\omega_{i}$ is the distance measurement noise bewteen UAV and anchor node $i$ which can be expressed as

$$
\omega_{i}=\left[\left(e_{D u}-e_{D i}\right)+\left(e_{A i}-e_{A u}\right)\right] c / 2 .
$$

Accordingly, the true value for the distance between UAV and anchor node $i$ is able to be calculated by the coordinates of these sensor nodes. Therefore, the following equation can be derived

$$
\left\{\begin{array}{c}
d_{1}=\left\|\boldsymbol{u}-\boldsymbol{u}_{1}\right\|+\omega_{1} \\
d_{2}=\left\|\boldsymbol{u}-\boldsymbol{u}_{2}\right\|+\omega_{2} \\
\vdots \\
d_{n}=\left\|\boldsymbol{u}-\boldsymbol{u}_{n}\right\|+\omega_{n}
\end{array},\right.
$$

where, $\boldsymbol{u}=[x, y, z]^{T}$ and $\boldsymbol{u}_{i}=\left[x_{i}, y_{i}, z_{i}\right]^{T}$ represent the position matrix of UAV and anchor node $i$. Then, by extending the equation into matrix form, we have

$$
\boldsymbol{D}=f(\boldsymbol{u})+\boldsymbol{\Omega} .
$$

Among the above equation, $\boldsymbol{D}, f(\boldsymbol{u})$ and $\Omega$ represent the measured distance information matrix, real distance information matrix and measurement noise matrix which are known as

$$
\begin{gathered}
\boldsymbol{D}=\left[d_{1}, d_{2}, \ldots, d_{n}\right]^{T}, \\
f(\boldsymbol{u})=\left[\tilde{d}_{1}, \tilde{d}_{2}, \ldots, \tilde{d}_{n}\right]^{T}, \\
\boldsymbol{\Omega}=\left[\omega_{1}, \omega_{2}, \ldots, \omega_{n}\right]^{T}=\left(\boldsymbol{C}_{0} \boldsymbol{E}+\boldsymbol{C}_{1} \boldsymbol{E}\right) c / 2,
\end{gathered}
$$

where, $\tilde{d}_{i}=\left\|\boldsymbol{u}-\boldsymbol{u}_{i}\right\|$ is the real distance between UAV and anchor node $i, \boldsymbol{E}=\left[e_{u}, e_{1}, e_{2}, \ldots, e_{n}\right]^{T}$ is defined as the measurement noise matrix, $C_{0}$ and $C_{1}$ are the coefficient matrix which can be written as

$$
\begin{gathered}
\boldsymbol{C}_{0}=\left[\begin{array}{ccccc}
-1 & 1 & 0 & \cdots & 0 \\
-1 & 0 & 1 & \cdots & 0 \\
\vdots & \vdots & \vdots & \ddots & \vdots \\
-1 & 0 & 0 & \cdots & 1
\end{array}\right]_{N \times(N+1)}, \\
\boldsymbol{C}_{1}=\left[\begin{array}{ccccc}
1 & -1 & 0 & \cdots & 0 \\
1 & 0 & -1 & \cdots & 0 \\
\vdots & \vdots & \vdots & \ddots & \vdots \\
1 & 0 & 0 & \cdots & -1
\end{array}\right]_{N \times(N+1)}
\end{gathered}
$$


Thus, the covariance matrix of $\Omega$ can be calculated as follows

$$
Q_{\Omega}=c\left(C_{0} Q_{E} C_{0}^{T}+C_{1} Q_{E} C_{1}^{T}\right)
$$

where, $Q_{E}$ is the covariance matrix of measurement noise matrix $\boldsymbol{E}$.

Finally, the likelihood function can be derived from the above-mentioned equations

$$
\begin{aligned}
p(\boldsymbol{D}, \boldsymbol{u})= & \frac{1}{(2 \pi)^{\frac{N}{2}} \operatorname{det}\left(\boldsymbol{Q}_{\boldsymbol{\Omega}}\right)^{\frac{1}{2}}} \\
& \cdot \exp \left[-\frac{1}{2}(\boldsymbol{D}-f(\boldsymbol{u}))^{T} \boldsymbol{Q}_{\boldsymbol{\Omega}}^{-1}(\boldsymbol{D}-f(\boldsymbol{u}))\right]
\end{aligned}
$$

\section{B. Maximum Likelihood Estimation}

Considering the existing characteristics, including the implementation simplicity, asymptotically unbiased and more efficiency [29], [30], the MLE approach is selected to estimate the position information of UAV. Through (14), the MLE of $\boldsymbol{u}$ can be obtained

$$
\hat{\boldsymbol{u}}=\arg \min \left[(\boldsymbol{D}-f(\boldsymbol{u}))^{T} \boldsymbol{Q}_{\boldsymbol{\Omega}}^{-1}(\boldsymbol{D}-f(\boldsymbol{u}))\right] .
$$

However, due to the nonlinearity of the real distance matrix $f(\boldsymbol{u})$, a closed-form solution for (15) does not exist. Therefore, a numerical minimisation method [29]- [33] will be introduced to approach the real solution.

Firstly, the estimation of $\boldsymbol{u}$ and the iteration factor at $j$ th iteration is defined as $\hat{\boldsymbol{u}}(j)$ and $\boldsymbol{\Delta}(j)$. It can be seen that

$$
\boldsymbol{u}=\hat{\boldsymbol{u}}(j)+\boldsymbol{\Delta}(j) .
$$

Thus, $f(\boldsymbol{u})$ can be linearised around $\hat{\boldsymbol{u}}(j)$, yielding

$$
f(\boldsymbol{u})=f(\hat{\boldsymbol{u}}(j))+G(\hat{\boldsymbol{u}}(j)) \boldsymbol{\Delta}(j),
$$

where, $G(\hat{\boldsymbol{u}}(j))$ is the Jacobian matrix

$$
G(\boldsymbol{u})=\frac{\partial f(\boldsymbol{u})}{\partial \boldsymbol{u}}
$$

From (9), the partial derivative of $f(\boldsymbol{u})$ with respect to $\boldsymbol{u}$ can be derived

$$
\frac{\partial f_{i}(\boldsymbol{u})}{\partial \boldsymbol{u}}=\frac{\partial \tilde{d}_{i}}{\partial \boldsymbol{u}}=\frac{\left(\boldsymbol{u}-\boldsymbol{u}_{i}\right)}{\left\|\boldsymbol{u}-\boldsymbol{u}_{i}\right\|}
$$

Substituting (19) into (18), the Jacobian matrix can be written as

$$
G(\boldsymbol{u})=\left[\frac{\left(\boldsymbol{u}-\boldsymbol{u}_{1}\right)}{\left\|\boldsymbol{u}-\boldsymbol{u}_{1}\right\|}, \frac{\left(\boldsymbol{u}-\boldsymbol{u}_{2}\right)}{\left\|\boldsymbol{u}-\boldsymbol{u}_{2}\right\|}, \ldots, \frac{\left(\boldsymbol{u}-\boldsymbol{u}_{n}\right)}{\left\|\boldsymbol{u}-\boldsymbol{u}_{n}\right\|}\right]^{T} .
$$

Combining (15) and (17), the estimation of the iteration factor can be calculated

$$
\begin{aligned}
\hat{\boldsymbol{\Delta}}(j)= & {\left[G^{T}(\hat{\boldsymbol{u}}(j)) \boldsymbol{Q}_{\boldsymbol{\Omega}}^{-1} G(\hat{\boldsymbol{u}}(j))\right]^{-1} } \\
& \cdot G^{T}(\hat{\boldsymbol{u}}(j)) \boldsymbol{Q}_{\boldsymbol{\Omega}}^{-1}(\boldsymbol{D}-\boldsymbol{f}(\hat{\boldsymbol{u}}(j))) .
\end{aligned}
$$

Finally, the estimate coordinate matrix of UAV at $(j+1)$ th iteration will be

$$
\hat{\boldsymbol{u}}(j+1)=\hat{\boldsymbol{u}}(j)+\hat{\Delta}(j)
$$

With the numerical minimisation method, the position information of UAV can be estimated. However, two additional problems, including the local minimum and the non-convergence problems which lead by the estimation method cannot be ignored. Clearly from (21), with a small $G^{T}(\hat{\boldsymbol{u}}(j)) \boldsymbol{Q}_{\boldsymbol{\Omega}}^{-1} G(\hat{\boldsymbol{u}}(j))$, a large oscillation for the iteration factor may occur. This may cause the estimation stopped at a local minimum or even the non-convergence for the estimator [29]- [32]. To prevent the aforementioned issue, an appropriate initial guess close to the true value is required. Therefore, considering the UAV is moving continuously, the previous estimation of the UAV position information will be served as the initial guess to avoid the iteration stopped at a local minimum, the non-convergence of the estimator and to reduce the iterations.

\section{Anchor Deployment Strategy}

Clearly from the positioning scheme and localisation equation shown in Fig.2 and (6), the number of anchor nodes in the system and their locations will have tremendous impact on the localisation performance. In addition, considering applications in extremely confined environments, such as a small storage tank or pressure vessel, which is hard to access, the anchor nodes can only be deployed near the entrance of the area inside that space. To be specific, the quantity and deployment area of anchor nodes are always limited in such environments. Consequently, the deployment strategies or layouts of the anchor nodes become particularly important to the localisation performance. Towards this end, the analysis on anchor deployment strategies to achieve high-precision localisation of UAV in extremely confined environments is carried out in this section.

In order to quantitatively analyse the localisation performance under different geometry configurations of anchor nodes, GDOP is introduced. GDOP is a significant metric to evaluate the precision of model parameters before positioning estimation [34]. It reflects the geometry configuration quality of anchor nodes. Here, suppose the covariance matrix of the unbiased estimation $\hat{\boldsymbol{u}}$ to be $\operatorname{Cov}(\hat{\boldsymbol{u}})$. Then GDOP can be defined as

$$
G D O P=\frac{\sqrt{\operatorname{tr}(\operatorname{Cov}(\hat{\boldsymbol{u}}))}}{\operatorname{co} \sigma_{e}},
$$

where, $\operatorname{tr}($.$) denotes matrix trace. The trace of the covariance$ matrix is related to the Cramér-Rao lower bound (CRLB) of the localisation algorithm.

According to the definition of CRLB, it is the inverse of the Fisher information matrix (FIM) [35]. Under Gaussian noise, FIM can be calculated through

$$
J(\boldsymbol{u})=G^{T}(\boldsymbol{u}) \boldsymbol{Q}_{\boldsymbol{\Omega}}^{-1} G(\boldsymbol{u}) .
$$

Thus, CRLB of the localisation algorithm can be represented as

$$
\bar{\sigma}_{\boldsymbol{u}}^{2}=\sum_{n=1}^{3}\left[J^{-1}(\boldsymbol{u})\right]_{n n},
$$

where, $\left[J^{-1}(\boldsymbol{u})\right]_{n n}$ denotes as the $n$th diagonal element of $J^{-1}(\boldsymbol{u})$.

Substituting (25) into (23), GDOP of the localisation algorithm can be computed by

$$
G D O P=\frac{\bar{\sigma}_{\boldsymbol{u}}}{c \sigma_{e}} .
$$



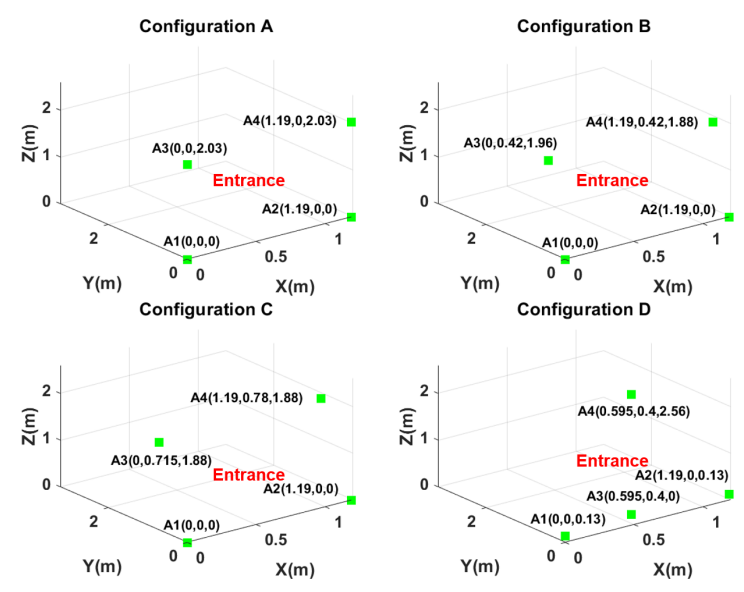

Fig. 3. Geometry configurations of anchor nodes.

To analyse the localisation performance under different anchor deployment strategies, four different types of geometry configurations for anchor nodes shown in Fig.3 are considered. The coordinates of each anchor node under different geometry configurations are listed in Table I, and the entrance of this confined space is supposed at the central area of $\mathrm{X}-\mathrm{Z}$ plane. The localisation area is set within a narrow space $(1.2 \mathrm{~m} \times$ $3.2 \mathrm{~m} \times 2.6 \mathrm{~m}$ ) which is the same as the actual experiment area in this paper, where $\mathrm{X}$-axis is set as the width direction, Y-axis is parallel to the depth direction, while $\mathrm{Z}$-axis denotes the altitude. Only four anchor nodes are considered in the simulation due to the difficulty for anchor deployment in the confined space. Moreover, considering the anchor nodes can only be deployed near the entrance of the confined space, the simulation will pay more attention to the performance change with the $\mathrm{Y}$ coordinate variation. Different $\mathrm{Y}$ coordinates of the target are taken into account for performance evaluation. In the simulation, the standard deviation (STD) of the measurement noise is set to be $0.1 \mathrm{~ns}$.

As shown in Fig.4, Fig.5, Fig.6 and Fig.7, GDOP of the algorithm under four different geometry configurations of anchor nodes with UAV at different position are simulated. From all the simulation results under different geometry configurations, it can be observed that with the increasing of the Y coordinate, the value of GDOP go up, which means a drop-off for the localisation accuracy. The performance dropoff is associated with the measurement noise of sensor nodes and the distribution of anchor nodes on the Y-axis. With the increasing distance between anchor nodes and UAV, the measurement noise will have greater impact on localisation accuracy. The accuracy descending rate is different for each geometry configuration. Configuration A holds the biggest accuracy descending rate compared with $\mathrm{B}$ and $\mathrm{C}$ with all the anchor nodes being deployed on the X-Z plane. From (20) and (24), FIM can be reshaped as

$$
F I M=\frac{1}{c^{2} \sigma_{\Omega}^{2}} \sum_{i=1}^{N} \frac{\left(\boldsymbol{u}-\boldsymbol{u}_{i}\right)}{\left\|u-u_{i}\right\|} \frac{\left(\boldsymbol{u}-\boldsymbol{u}_{i}\right)^{T}}{\left\|u-u_{i}\right\|} .
$$

Considering the anchor nodes are all deployed on the $\mathrm{X}-\mathrm{Z}$
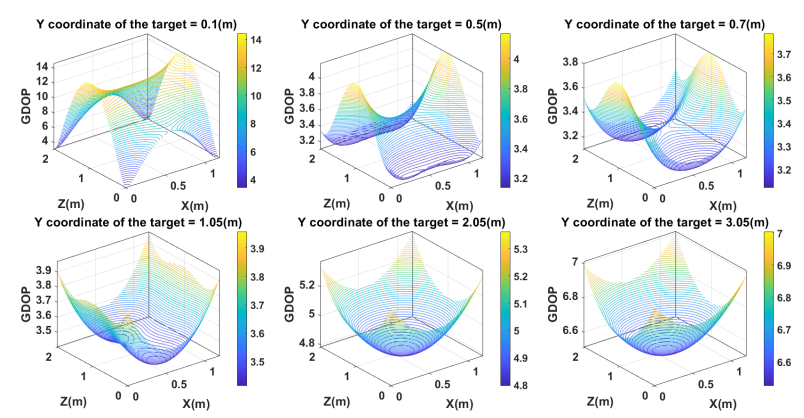

Fig. 4. GDOP under configuration $\mathrm{A}$ of anchor nodes.
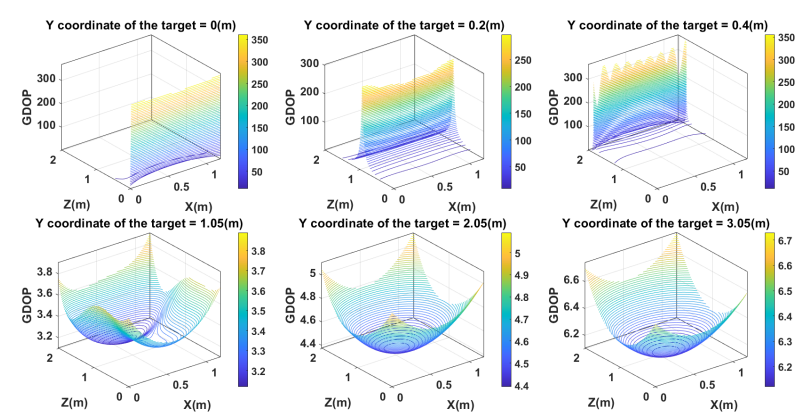

Fig. 5. GDOP under configuration B of anchor nodes.

plane, when UAV is on the same plane, FIM will be singular. This means no unbiased estimator for $\boldsymbol{u}$ exists with a finite variance [27] and the algorithm will lose its effectiveness. Moreover, it appears that GDOP becomes larger with UAV close to the $\mathrm{X}-\mathrm{Z}$ plane, especially for the centre of the plane under configuration $\mathrm{A}$. For configuration $\mathrm{B}$, where anchor 3 and anchor 4 are deployed a little deeper in the area with the same $\mathrm{Y}$ coordinate, the accuracy descending rate successfully decreased as shown in Fig.5. GDOP becomes larger when UAV is approaching the plane composed of four anchor nodes. Clearly, within the composed plane, Y coordinate of UAV is able to be expressed linearly by $\mathrm{X}$ and $\mathrm{Z}$ coordinates. Thus, the inverse matrix of FIM does not exist, which will result in the lose effectiveness of the algorithm. Similar to configuration B, anchor 3 and anchor 4 are also deployed a little deeper in the area, but with the different $\mathrm{Y}$ coordinate for configuration $\mathrm{C}$. As apparent from Fig.6, the accuracy is further improved with the increasing of the $\mathrm{Y}$ coordinate in contrast to configuration B. Whereas, the precision degradation still exists when UAV is close to the planes composed of any three anchor nodes. Completely different from all the aforementioned deployment strategies, two anchor nodes are deployed on the top and bottom of the confined space for configuration $\mathrm{D}$ which is analysed in Fig.7. Localisation performance becomes worse with the increasing of the $\mathrm{Z}$ coordinate under different $\mathrm{Y}$ coordinates, associated with the small distribution of anchor nodes on Z-direction.

To further evaluate the localisation performance under different deployment strategies, RMSE of the algorithm under these four geometry configurations are given in Fig.8, Fig.9, Fig.10 and Fig.11. Clearly, RMSE becomes larger with the 
TABLE I

COORDINATES OF ANCHOR NODES UNDER DIFFERENT GEOMETRY CONFIGURATIONS

\begin{tabular}{|l|l|l|l|l|}
\hline Geometry Configuration & Anchor 1 & Anchor 2 & Anchor 3 & Anchor 4 \\
\hline Configuration A & $(0,0,0) \mathrm{m}$ & $(1.19,0,0) \mathrm{m}$ & $(0,0,2.03) \mathrm{m}$ & $(1.19,0,2.03) \mathrm{m}$ \\
Configuration B & $(0,0,0) \mathrm{m}$ & $(1.19,0,0) \mathrm{m}$ & $(0,0.42,1.96) \mathrm{m}$ & $(1.19,0.42,1.88) \mathrm{m}$ \\
Configuration C & $(0,0,0) \mathrm{m}$ & $(1.19,0,0) \mathrm{m}$ & $(0,0.715,1.88) \mathrm{m}$ & $(1.19,0.78,1.88) \mathrm{m}$ \\
Configuration D & $(0,0,0.13) \mathrm{m}$ & $(1.19,0,0.13) \mathrm{m}$ & $(0.595,0.4,0) \mathrm{m}$ & $(0.595,0.4,2.56) \mathrm{m}$ \\
\hline
\end{tabular}
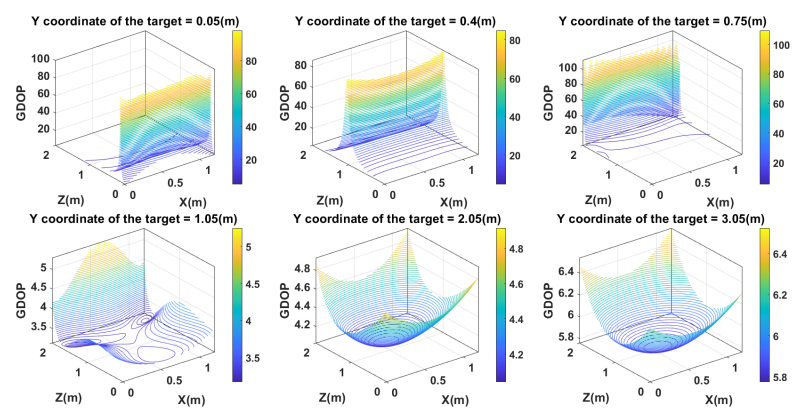

Fig. 6. GDOP under configuration $\mathrm{C}$ of anchor nodes.
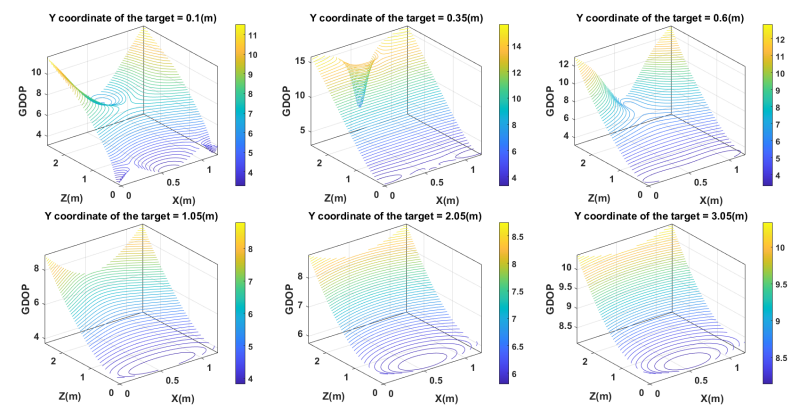

Fig. 7. GDOP under configuration D of anchor nodes.

increasing of the $\mathrm{Y}$ coordinate, and configuration A holds the biggest descending rate of localisation accuracy compared with $\mathrm{B}$ and $\mathrm{C}$. For configuration $\mathrm{A}$, the same result can be obtained that when UAV is close to the $\mathrm{X}-\mathrm{Z}$ plane, especially the centre of the plane, there will be a sharp drop-off for the accuracy. When UAV flies away from the plane, the influence will be eased. On the other hand, considering the RMSE distribution under configuration B and C. It appears that with UAV flying within the area composed of these four anchor nodes, there will be performance oscillation which has the same conclusion in contrast to the analysis on GDOP. The localisation performance is better than configuration A when out of the area constituted by these anchor nodes. Finally, from Fig.11, it is clear to find that, even an accurate result can be obtained with a small $\mathrm{Z}$ coordinate for configuration $\mathrm{D}$, the overall accuracy is kept at a low level in contrast to the other three geometry configurations.

\section{EXPERIMENTS}

\section{A. Experiment Setup}

To evaluate the localisation performance under these four geometry configurations of anchor nodes in actual environ-

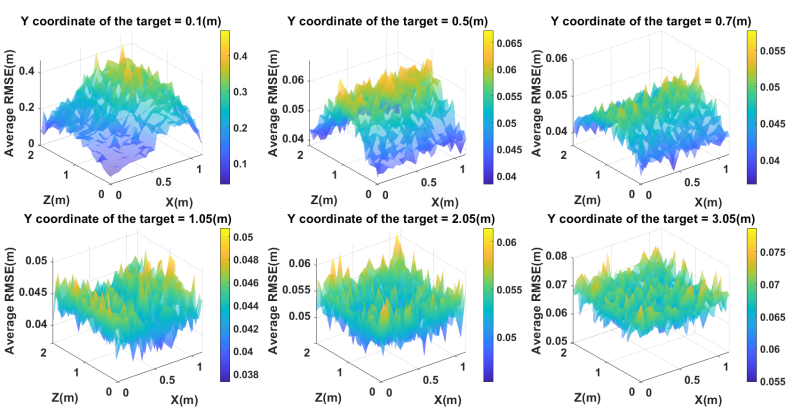

Fig. 8. RMSE under configuration $A$ of anchor nodes.

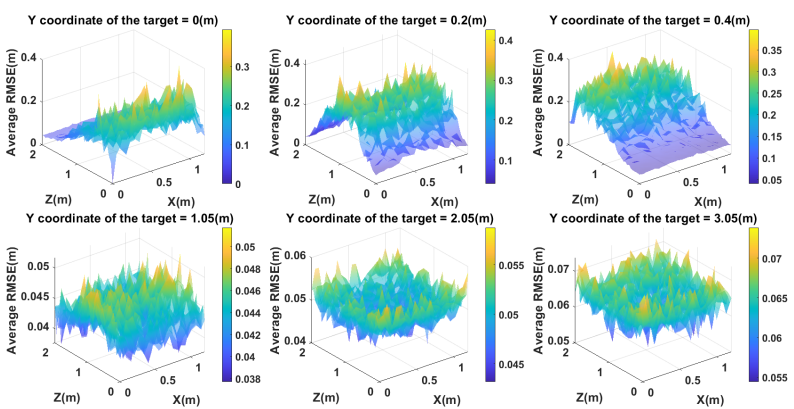

Fig. 9. RMSE under configuration B of anchor nodes.

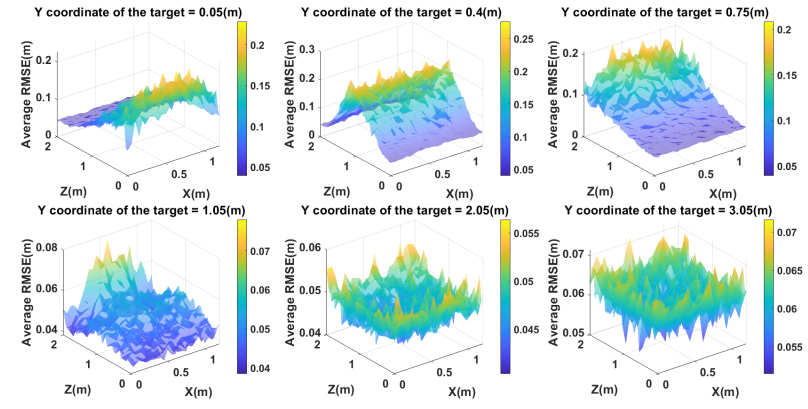

Fig. 10. RMSE under configuration $\mathrm{C}$ of anchor nodes.

ment, experiments are performed with a hybrid UAV localisation system consisting of UWB based sensor nodes designed by Pozyx and a commercial low-cost mini quadcopter - DJI Tello EDU. For the UWB based sensor nodes, considering the stability and accuracy of the localisation performance, channel 2 is selected which is defined by the IEEE 802.15.4 standard [36]. The centre frequency is $3993.6 \mathrm{MHz}$ and the bandwidth is 499.2 MHz. The localisation area is set within a narrow space $(1.2 \mathrm{~m} \times 3.2 \mathrm{~m} \times 2.6 \mathrm{~m})$ in lab environment with four anchor nodes existing as depicted in Fig.12. The UAV 

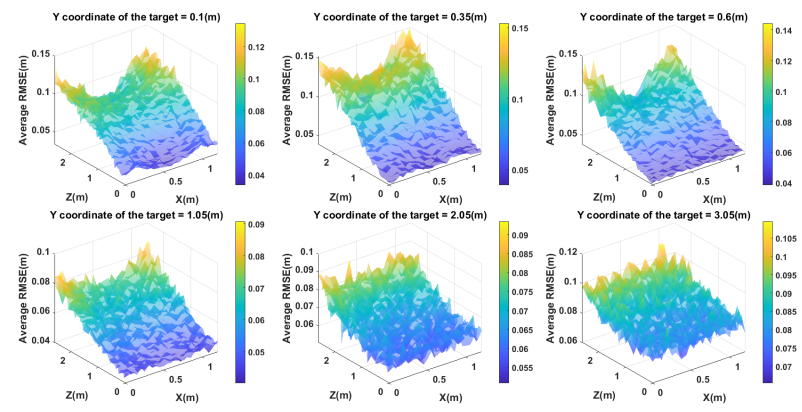

Fig. 11. RMSE under configuration D of anchor nodes.

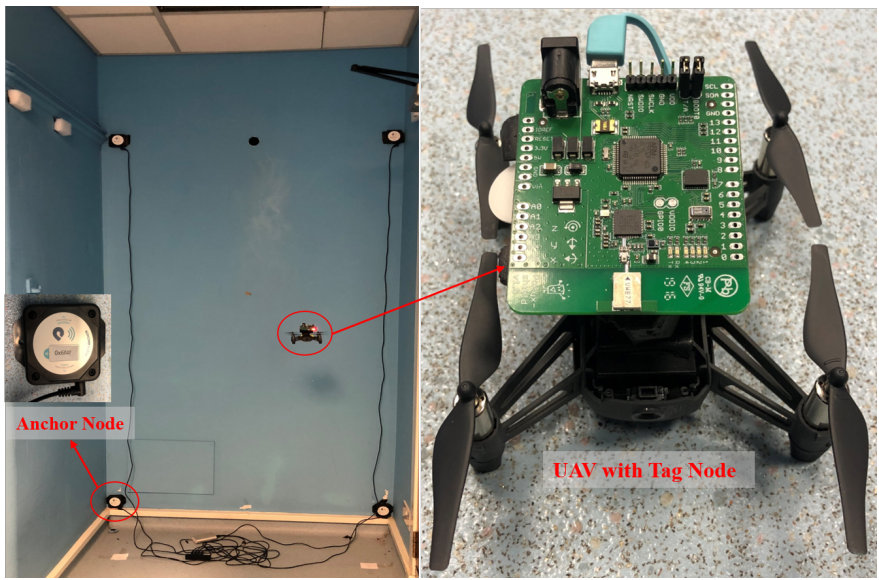

Fig. 12. Lab experiment environment and UAV.
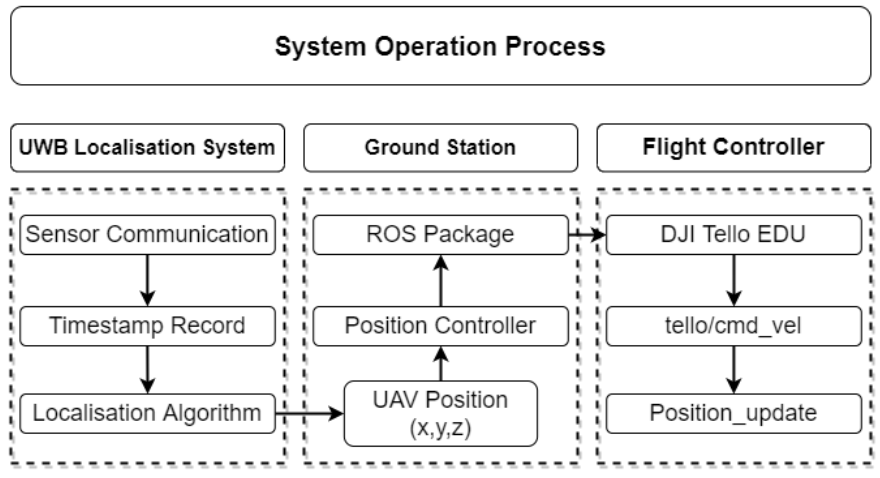

Fig. 13. System operation process.

localisation system has three main modules: UWB based localisation module, ground station (localisation server) and UAV. The system structure and diagrams of each module are given in Fig.1 and Fig.13. During the localisation process, a position request will be sent out from the tag node equipped on UAV. Afterwards, the recorded information from the communication between sensor nodes will be transmitted to the ground station (localisation server) for position estimation. The localisation server and ground station is a laptop where the localisation algorithm and position controller are implemented. With the estimated position information, the response command will be given by the controller and encapsulated as a ROS package. Finally, the package will be transmitted to UAV for position update.

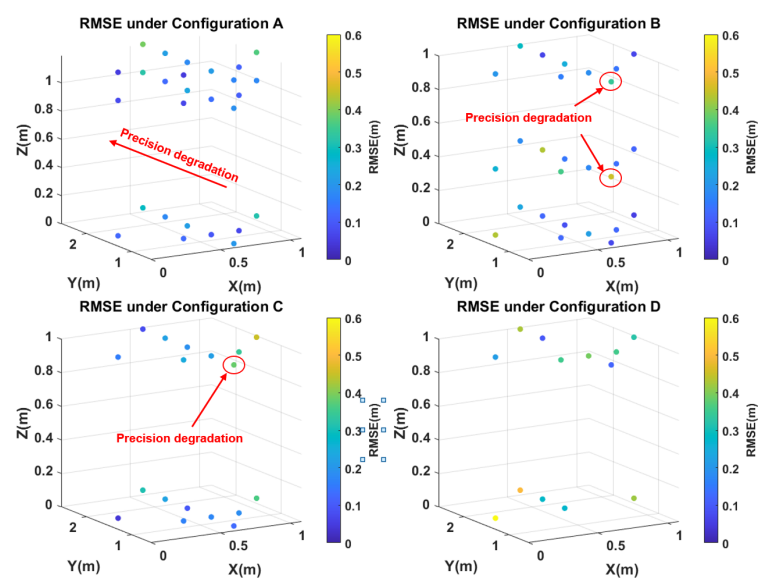

Fig. 14. RMSE under different geometry configurations of anchor nodes.

\section{B. Localisation Performance Evaluation}

In order to validate the localisation performance comprehensively, the experiments are divided into two parts, the static experiments and flight tests. Firstly, the performance testing was done at some representative points statically without UAV flying under different anchor deployment strategies. These experiments pay more attention to analyse the impact of different anchor deployment strategies on localisation performance in actual environments. The true location of each test point was measured manually. For each test point, 100 measurements were repeated to eliminate the influence of unreasonable values on positioning. The RMSE and STD of the 100 RMSE for each test point under four geometry configurations of anchor nodes are presented in Fig.14 and Fig.15. Where X(m), Y(m) and $\mathrm{Z}(\mathrm{m})$ represent the coordinate of the test points, the colour bar is denoted as the distribution of average RMSE and the STD of RMSE. Moreover, the cumulative distribution function (CDF) of the localisation error at four representative test points under different geometry configurations are shown in Fig.16. The median and 95th percentile localisation errors are given in Table II.

From the results, it can be found out that, high-accuracy result with an average RMSE under $0.15 \mathrm{~m}$ can be obtained with Y coordinate of the target within $0.5 \mathrm{~m}-2 \mathrm{~m}$ for configuration A. The average STD of RMSE keeps below $0.07 \mathrm{~m}$ which also means a high-precision performance. However, with the $\mathrm{Y}$ coordinate larger than $2 \mathrm{~m}$, average RMSE is increased to $0.26 \mathrm{~m}$ which is significantly inferior to configuration $\mathrm{B}$ and C. For configuration B and C, with the same $\mathrm{Y}$ coordinate, the accuracy is improved with the average RMSE less than $0.23 \mathrm{~m}$ and $0.21 \mathrm{~m}$. Whereas, it appears that when the target is near the plane consisting of anchor nodes, the violent oscillation can be observed in Fig.14 and Fig.15 with the average RMSE and STD larger than $0.35 \mathrm{~m}$. But the average RMSE and STD are still kept below $0.2 \mathrm{~m}$ and $0.07 \mathrm{~m}$ for most test points with configuration $B$ and C. Particularly, the STD of RMSE for configuration $\mathrm{C}$ is even lower than $0.04 \mathrm{~m}$ excluding the test points aforementioned. Moreover, it is clear to find out that 


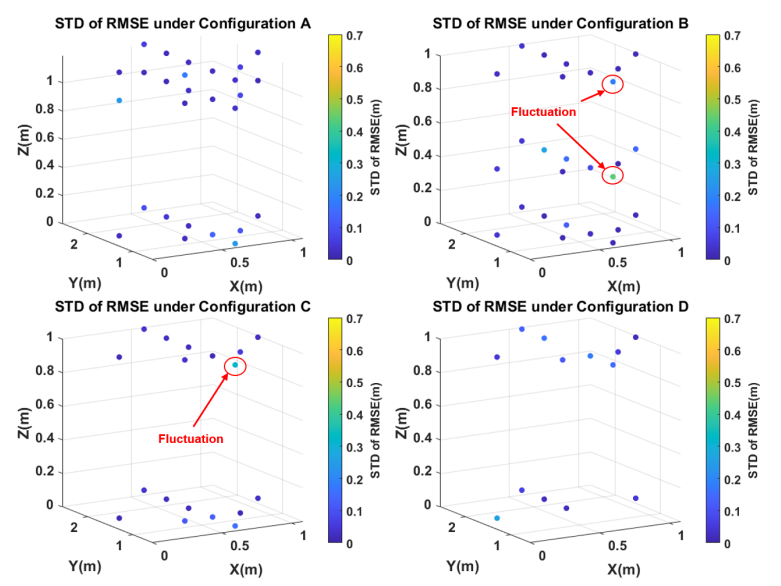

Fig. 15. STD of RMSE under different geometry configurations of anchor nodes.
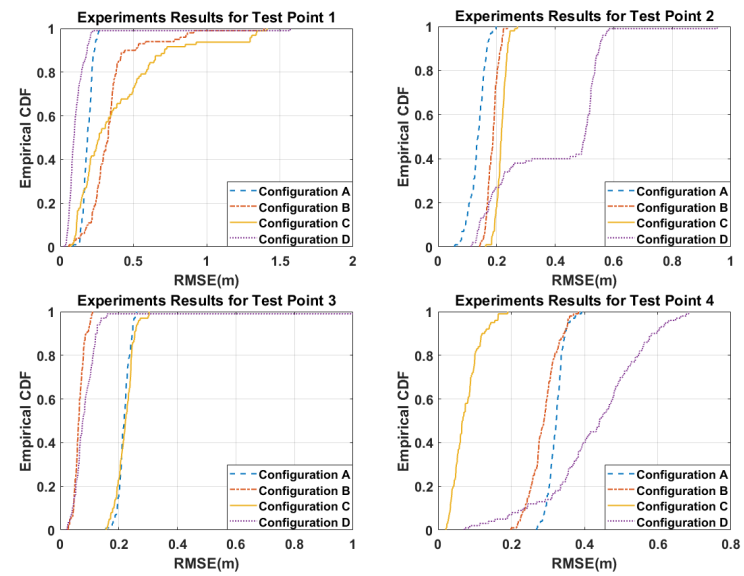

Fig. 16. CDF for representative test points under different geometry configurations of anchor nodes. Coordinate of test point 1 : $[0.59,0.550,0.99] \mathrm{m}$, test point 2: $[0.59,1.050,0.99] \mathrm{m}$, test point 3 : $[0.59,2.050,0.99] \mathrm{m}$ and test point $4:[0.59,2.550,0.99] \mathrm{m}$.

configuration D holds the worst performance with the average RMSE larger than $0.3 \mathrm{~m}$.

On the other hand, from Table II and Fig.16, it appears that for test point 1 and 2 with a smaller $\mathrm{Y}$ coordinate $(0.550 \mathrm{~m}$ and $1.050 \mathrm{~m}$ ), configuration A holds the great performance with median error less than $0.19 \mathrm{~m}$. However, with the increased $\mathrm{Y}$ coordinate for test point $3(2.050 \mathrm{~m})$ and test point $4(2.550$ $\mathrm{m})$, the median error and 95th percentile error are all increased. Another key observation is that for test point 3 and test point 4, configuration $\mathrm{B}$ and $\mathrm{C}$ can achieve higher accuracy. Especially for configuration $\mathrm{C}$ at test point 4 , the localisation error is significantly decreased with a $0.07 \mathrm{~m}$ median error and $0.15 \mathrm{~m}$ 95th percentile error. Nevertheless, the performance oscillation can be observed for these two geometry configurations with the target near the entrance of the confined environment at test point 1 . Interestingly, for test point 1 , configuration D holds the best performance with the median error and 95th percentile error to be $0.09 \mathrm{~m}$ and $0.20 \mathrm{~m}$. Whereas, the localisation performance is unstable with the STD larger than $0.13 \mathrm{~m}$ for
TABLE II

LOCALISATION ERROR FOR REPRESENTATIVE TEST POINTS

\begin{tabular}{|l|l|l|l|}
\hline Geometry Configuration & Median Error $(\mathrm{m})$ & $\begin{array}{l}\text { 95th Percentile } \\
\text { Error }(\mathrm{m})\end{array}$ & STD (m) \\
\hline Configuration A-Test 1 & 0.19 & 0.24 & 0.03 \\
Configuration A-Test 2 & 0.14 & 0.17 & 0.03 \\
Configuration A-Test 3 & 0.22 & 0.25 & 0.02 \\
Configuration A-Test 4 & 0.32 & 0.35 & 0.02 \\
Configuration B-Test 1 & 0.33 & 0.78 & 0.18 \\
Configuration B-Test 2 & 0.19 & 0.22 & 0.02 \\
Configuration B-Test 3 & 0.06 & 0.10 & 0.02 \\
Configuration B-Test 4 & 0.29 & 0.35 & 0.04 \\
Configuration C-Test 1 & 0.27 & 1.30 & 0.31 \\
Configuration C-Test 2 & 0.22 & 0.24 & 0.02 \\
Configuration C-Test 3 & 0.22 & 0.27 & 0.03 \\
Configuration C-Test 4 & 0.07 & 0.15 & 0.04 \\
Configuration D-Test 1 & 0.09 & 0.20 & 0.15 \\
Configuration D-Test 2 & 0.50 & 0.56 & 0.18 \\
Configuration D-Test 3 & 0.08 & 0.13 & 0.17 \\
Configuration D-Test 4 & 0.44 & 0.62 & 0.13 \\
\hline
\end{tabular}

Test $1=[0.59,0.550,0.99] \mathrm{m}$, Test $2=[0.59,1.050,0.99] \mathrm{m}$, Test $3=$ $[0.59,2.050,0.99] \mathrm{m}$, Test $4=[0.59,2.550,0.99] \mathrm{m}$

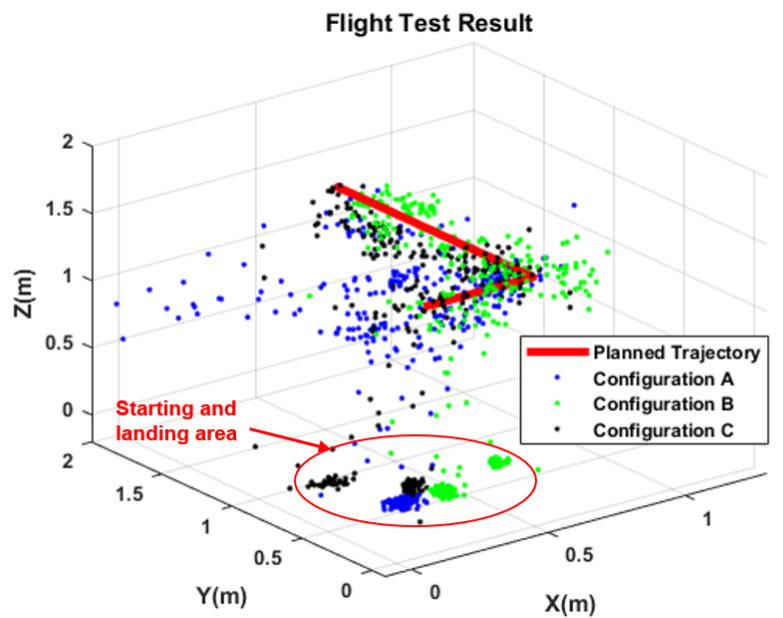

Fig. 17. Localisation results of the flight tests.

all the test points under configuration D. Meanwhile, for test point 2 and test point 4 , the median errors are increased to 0.50 $\mathrm{m}$ and $0.44 \mathrm{~m}$ which is unacceptable for UAV positioning in extremely confined environments.

To further evaluate the localisation performance and illustrate the effectiveness of the proposed approach, flight tests under different geometry configurations of anchor nodes were carried out with UAV flying along a planned trajectory. The planned trajectory with the estimated position under different geometry configurations of anchor nodes is given in Fig.17. Therein, the flight tests were only performed with configuration $\mathrm{A}, \mathrm{B}$ and $\mathrm{C}$, considering the unacceptable and unstable accuracy for configuration D. As apparent from the localisation results, the same conclusion can be made in contrast to the results from simulation and static experiments.

In conclusion, from the experimental results, it can be determined that in the space with a small size in depth direction (less than $2 \mathrm{~m}$ ), configuration $\mathrm{A}$ is able to provide precise and accurate localisation for UAV with the average RMSE and STD under $0.15 \mathrm{~m}$ and $0.07 \mathrm{~m}$, respectively. On 
the contrary, configuration $\mathrm{B}$ and $\mathrm{C}$ are much more suitable to be applied in the area with a larger size in depth direction, especially for configuration $\mathrm{C}$ where higher accuracy can be provided. However, performance drop-off will be noticed when the UAV is approaching the entrance of the area (near the anchor nodes deployment area).

For our focused application scenarios in extremely confined environments, one of the most important indexes for UAV positioning is the STD for the localisation error. This is also known as the precision or the level of performance oscillation for the system. The precision will have a huge impact on the stability of UAV in such environments. Considering the dimensions of the DJI Tello $(98 \mathrm{~mm} \times 92.5 \mathrm{~mm} \times 41 \mathrm{~mm})$, the precision less than $0.098 \mathrm{~m}$ is acceptable for our application which can be attained with the suitable geometry configuration of anchor nodes. On the other hand, regarding to the absolute accuracy, this index is relevant to the situation that whether UAV can fly along with the planned trajectory and hit the targeted points or not. Owing to the air flow disturbance, in such environments, UAV cannot fly too close to the wall for inspection, in case of the instability control or crash down. Therefore, a safety distance is required when planning the trajectory or target points. Meanwhile, the system is developed for smart inspection, even UAV cannot hit every target point accurately, the camera can still record the video or picture in that area for further inspection. Thus, the average RMSE less than $0.2 \mathrm{~m}$ is acceptable for our application. In conclusion, with our proposed approach, the precise UAV positioning can be achieved in extremely confined environments under different circumstances.

\section{CONCLUSION}

Under the consideration of the increasing demand on UAV autonomous inspection, especially for the inspection in the small storage tank or pressure vessel which is hard or impossible for humans to access, this paper proposed a high-precision UWB based UAV positioning approach for applications in extremely confined environments. Firstly, the TW-TOF based localisation scheme with the MLE method was combined as the localisation algorithm for precise UAV positioning. Then, the anchor deployment strategies were analysed and simulated with the considerations on the highly restricted number and deployment area of anchor nodes in the confined environments. This was done to find the most suitable geometry configurations with accurate and robust performance. Finally, the realworld tests were carried out to validate the performance of the proposed approach. As shown in the results, the approach could provide precise 3D UAV positioning with a decimetrelevel accuracy and centimetre-level precision. This was done under three different geometry configurations of anchor nodes.

\section{REFERENCES}

[1] L. Yu, E. Yang, P. Ren, C. Luo, G. Dobie, D. Gu, and X Yan, "Inspection robots in oil and gas industry: a review of current solutions and future trends," in Proc. IEEE 25th Int. Conf. Automat. Comput. (ICAC), Lancaster, UK, Sep. 2019, pp. 1-6.

[2] R. La Scalea, M. Rodrigues, D. P. M. Osorio, C. H. Lima, R. D. Souza, H. Alves, and K. C. Branco, "Opportunities for autonomous UAV in harsh environments," in Proc. 16th Int. Symp. Wireless Commun. Syst. (ISWCS), Oulu, Finland, Aug. 2019, pp. 227-232.
[3] Flyability. Elios 2-Indoor Drone for Confined Space Inspections. [Online]. Available: https://www.flyability.com/elios-2, Accessed on: Oct.21, 2021.

[4] A. Couturier, and M. Akhloufi, "A review on absolute visual localization for UAV," Robot. Auto. Syst., vol. 135, pp. 103666, Jan. 2021.

[5] S. Krul, C. Pantos, M. Frangulea, and J. Valente, "Visual SLAM for Indoor Livestock and Farming Using a Small Drone with a Monocular Camera: A Feasibility Study,” Drones, vol. 5, no. 2, pp. 41, May. 2021

[6] R. Y. Brogaard, M. Zajaczkowski, L. Kovac, O. Ravn, and E. Boukas, "Towards UAV-Based Absolute Hierarchical Localization in Confined Spaces," in Proc. IEEE Int. Symp. Safety, Secur., Rescue Robot. (SSRR), Nov. 2020, pp. 182-188.

[7] F. Wang, K. Wang, S. Lai, S. K. Phang, B. M. Chen, and T. H. Lee, "An efficient UAV navigation solution for confined but partially known indoor environments," in Proc. 11th IEEE Int. Conf. Control Autom. (ICCA), Taichung, Taiwan, Jun. 2014, pp. 1351-1356.

[8] P. Tripicchio, M. Satler, M. Unetti, and C. A. Avizzano, "Confined spaces industrial inspection with micro aerial vehicles and laser range finder localization," Int. J. Micro Air Veh., vol. 10, no. 2, pp. 207-224, 2018.

[9] S. Lee, D. Har, and D. Kum, "Drone-assisted disaster management: Finding victims via infrared camera and lidar sensor fusion," in Proc. 3rd Asia-Pacific World Congr. Comput. Sci. Eng. (APWC CSE), Nadi, Fiji, Dec. 2016, pp. 84-89.

[10] G. A. Kumar, A. K. Patil, R. Patil, S. S. Park, and Y. H. Chai, "A LiDAR and IMU Integrated Indoor Navigation System for UAVs and Its Application in Real-Time Pipeline Classification," Sensors., vol. 17, no. 6, pp. 1268, 2017.

[11] A. R. Castaño, Romero, H., Capitán, J., Andrade, J. L., and Ollero, A, "Development of a Semi-autonomous Aerial Vehicle for Sewerage Inspection," in Proc. Robot 2017: 3rd Iberian Robot. Conf., Seville, Spain, Nov. 2017, pp. 75-86.

[12] A. Alsayed, A. Yunusa-Kaltungo, M. K. Quinn, F. Arvin, and M. R. A. Nabawy, "Drone-Assisted Confined Space Inspection and Stockpile Volume Estimation," Remote Sensing, vol. 13, no. 17, pp. 3356, Aug. 2021.

[13] H. Lücken, "Communication and localization in UWB sensor networks: A synergetic approach,” Ph.D. dissertation, ETH Zürich., Zürich, Switzerland, 2012.

[14] A. A. D'Amico, U. Mengali, and L. Taponecco, "TOA estimation with the IEEE 802.15. 4a standard," IEEE Trans. Wireless Commun., vol. 9, no. 7, pp. 2238-2247, Jul. 2010.

[15] N. H. Nguyen and K. Dogancay, "Optimal geometry analysis for multistatic TOA localization,” IEEE Trans. Signal Process., vol. 64, no. 16, pp. 4180-4193, Aug. 2016.

[16] J. Tiemann and C. Wietfeld, "Scalable and precise multi-uav indoor navigation using tdoa-based uwb localization," in Proc. Int. Conf. Indoor Positioning Indoor Navigat., Sapporo, Japan, Sep. 2017, pp. 1-7.

[17] J. L. Rullán-Lara, S. Salazar, and R. Lozano, "Real-time localization of an UAV using Kalman filter and a Wireless Sensor Network," J. Intell. Robot. Syst., vol. 65, no. 1-4, pp. 283-293, Jan. 2012.

[18] M. N. de Sousa and R. S. Thoma, "Localization of UAV in urban scenario using multipath exploiting TDoA fingerprints," in Proc. IEEE 29th Annu. Int. Symp. Pers., Indoor Mobile Radio Commun. (PIMRC), Bologna, Italy, Sep. 2018, pp. 1394-1399.

[19] A. Benini, A. Mancini, and S. Longhi, "An imu/uwb/vision-based extended kalman filter for mini-uav localization in indoor environment using 802.15. 4a wireless sensor network," J. Intell. Robot. Syst., vol. 70, no. 1-4, pp. 461-476, Apr. 2013.

[20] K. Guo, Z. Qiu, C. Miao, A. H. Zaini, C.-L. Chen, W. Meng, and L. Xie, "Ultra-wideband-based localization for quadcopter navigation," Unmanned Syst., vol. 4, no. 1, pp. 23-34, 2016.

[21] J. Li, Y. Bi, K. Li, K. Wang, F. Lin, and B. M. Chen, "Accurate 3d localization for mav swarms by uwb and imu fusion," in Proc. IEEE Int. Conf. Control Autom. (ICCA), Anchorage, AK, USA, Jun. 2018, pp. 100-105.

[22] T. M. Nguyen, A. H. Zaini, K. Guo, and L. Xie, "An ultra-widebandbased multi-UAV localization system in GPS-denied environments," in Proc. Int. Micro Air Vehicles Conf. (IMAV), Beijing, China, Oct. 2016, pp. 56-61.

[23] W. You, F. Li, L. Liao, and M. Huang, "Data fusion of UWB and IMU based on unscented Kalman filter for indoor localization of Quadrotor UAV," IEEE Access., vol. 8, pp. 64971-64981, 2020.

[24] F. Lazzari, A. Buffi, P. Nepa, and S. Lazzari, "Numerical investigation of an UWB localization technique for unmanned aerial vehicles in outdoor scenarios," IEEE Sensors J., vol. 17, no. 9, pp. 2896-2903, May. 2017. 
[25] M.-G. Li, H. Zhu, S.-Z. You, and C.-Q. Tang, "UWB-Based Localization System Aided With Inertial Sensor for Underground Coal Mine Applications," IEEE Sensors J., vol. 20, no. 12, pp. 6652-6669, Feb. 2020.

[26] M. A. Spirito, "On the accuracy of cellular mobile station location estimation," IEEE Trans. Veh. Technol., vol. 50, no. 3, pp. 674-685, May., 2001.

[27] A. N. Bishop, B. Fidan, B. D. O. Anderson, K. Dogcancay, and P. N. Pathirana, "Optimality analysis of sensor-target localization geometries," Automatica., vol. 46, no. 3, pp. 479-492, May., 2010.

[28] S. Gezici et al., "Localization via ultra-wideband radios: a look at positioning aspects for future sensor networks," IEEE Signal Process. Mag., vol. 22, no. 4, pp. 70-84, Jul., 2005.

[29] S. M. Kay, Fundamentals of Statistical Signal Processing: Estimation Theory. Upper Saddle River, NJ, USA: Prentice-Hall, 1993.

[30] T. Li, A. Ekpenyong, and Y.-F. Huang, "Source localization and tracking using distributed asynchronous sensors," IEEE Trans. Signal Process., vol. 54, no. 10, pp. 3991-4003, Oct. 2006.

[31] H. Xiong, Z. Chen, W. An, and B. Yang, "Robust TDOA localization algorithm for asynchronous wireless sensor networks," Int. J. Distrib. Sensor Netw., vol. 11, no. 5, pp. 10, May. 2015.

[32] T. Wang, H. Ding, H. Xiong, and L. Zheng, "A compensated multianchors TOF-based localization algorithm for asynchronous wireless sensor networks," IEEE Access., vol. 7, pp. 64162-64176, 2019.

[33] J. Smith and J. Abel, "Closed-form least-squares source location estimation from range-difference measurements," IEEE Trans. Acoust., Speech, Signal Process., vol. ASSP-35, no. 12, pp. 1661-1669, Dec. 1987.

[34] S. Li, G. Li, L. Wang, Y. Zhou, Y. Peng, and J. Fu, "A three-dimensional robust ridge estimation positioning method for UWB in a complex environment," Adv. Space Res., vol. 60, no. 12, pp. 2763-2775, Dec. 2017.

[35] H. Poor, An Introduction to Signal Detection and Estimation. 2nd ed. New York, NY, USA: Springer, 1994.

[36] "IEEE Standard for Low-Rate Wireless Networks," in IEEE Std 802.15.4-2015 (Revision of IEEE Std 802.15.4-2011) , pp.1-709, 22 April 2016, doi: 10.1109/IEEESTD.2016.7460875.

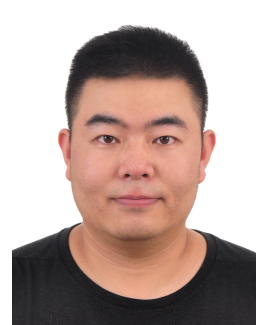

Beiya Yang received the B.Eng. degree in Electronic Information Engineering from Northwestern Polytechnical University, Xi'an, China, in 2013 and the M.Sc degree in Information and Communication Engineering from National University of Defense Technology, Changsha, China, in 2015. He is currently pursuing the Ph.D. degree in the Department of Design, Manufacturing and Engineering Management (DMEM) at University of Strathclyde, Glasgow, UK, since 2019. His current research interests include indoor localisation technology, unmanned aerial vehicles (UAV) localisation and wireless sensor networks.

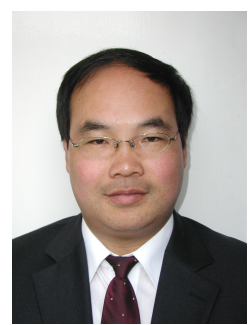

Erfu Yang (IEEE Member, 2005) received his Ph.D. degree in Robotics from the School of Computer Science and Electronic Engineering, the University of Essex, Colchester, UK, in 2008. $\mathrm{He}$ is currently a Senior Lecturer in the Department of Design, Manufacturing and Engineering Management (DMEM), the University of Strathclyde, Glasgow, UK. His main research interests include robotics, autonomous systems, mechatronics, manufacturing automation, signal and image processing, computer vision and applications of machine learning and artificial intelligence, etc. He has over 145 publications in these areas, including more than 70 journal papers and 10 book chapters. Dr. Yang has been awarded over 15 research grants as $\mathrm{PI}$ (principal investigator) or $\mathrm{Cl}$ (co-investigator). $\mathrm{He}$ is the Fellow of the UK Higher Education Academy, member of IEEE and IEEE Society of Robotics and Automation, and the IET SCOTLAND Manufacturing Technical Network. He is also an associate editor for the Cognitive Computation journal published by Springer.

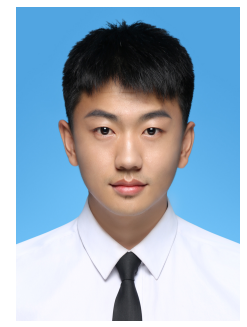

Leijian Yu received the B.Eng. degree in electrical information engineering and the M.Sc degree in information and communication engineering from China University of Petroleum (East China), Qingdao, China, in 2015 and 2018, respectively. $\mathrm{He}$ is currently pursuing the Ph.D. degree in the Department of Design, Manufacturing and Engineering Management (DMEM) at the University of Strathclyde, Glasgow, UK, since 2018. His current research interests include machine learning with applications on unmanned aerial vehicles (UAV), UAV vision-based autonomous navigation, and image contrast enhancement.

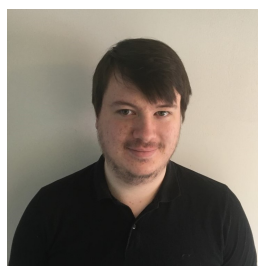

Andrew Loeliger received a B.Eng degree in Electronic \& Electrical Engineering in 2019 and an M.Sc in Mechatronics \& Automation in 2020, both from the University of Strathclyde, Glasgow, Scotland. $\mathrm{He}$ is currently pursuing a Ph.D in the Department of Design, Manufacturing and Engineering Management (DMEM) at the University of Strathclyde since 2020. His current research interests include cobot arm control, multiple autonomous robot (MAR) development and additive sensor systems. 